\title{
Survival kinase genes present prognostic significance in glioblastoma
}

\author{
Robin T. Varghese ${ }^{1}$, Yanping Liang ${ }^{1}$, Ting Guan ${ }^{2}$, Christopher T. Franck ${ }^{1,2}$, Deborah \\ F. Kelly ${ }^{1,3,4,6}$, Zhi Sheng ${ }^{1,4,5,6}$ \\ ${ }^{1}$ Virginia Tech Carilion Research Institute, Roanoke, VA 24016, USA \\ ${ }^{2}$ Laboratory for Interdisciplinary Statistical Analysis, Department of Statistics, Virginia Tech, Blacksburg, VA 24061, USA \\ ${ }^{3}$ Department of Biological Sciences, College of Sciences at Virginia Tech, Blacksburg, VA 24061, USA \\ ${ }^{4}$ Department of Internal Medicine, Virginia Tech Carilion School of Medicine, Roanoke, VA 24016, USA \\ ${ }^{5}$ Department of Biological Sciences and Pathobiology, Virginia-Maryland College of Veterinary Medicine, Virginia Tech, \\ Blacksburg, VA 24061, USA \\ ${ }^{6}$ Faculty of Health Science, Virginia Tech, Blacksburg, VA 24061, USA
}

Correspondence to: Zhi Sheng, e-mail: zhisheng@vtc.vt.edu

Keywords: survival kinase genes, glioblastoma, tumor recurrence, prognosis, PIK3CB

Received: December 22, 2015

Accepted: February 14, 2016

Published: March 04, 2016

\section{ABSTRACT}

Cancer biomarkers with a strong predictive power for diagnosis/prognosis and a potential to be therapeutic targets have not yet been fully established. Here we employed a loss-of-function screen in glioblastoma (GBM), an infiltrative brain tumor with a dismal prognosis, and identified 20 survival kinase genes (SKGs). Survival analyses using The Cancer Genome Atlas (TCGA) datasets revealed that the expression of CDCP1, CDKL5, CSNK1E, IRAK3, LATS2, PRKAA1, STK3, TBRG4, and ULK4 stratified GBM prognosis with or without temozolomide (TMZ) treatment as a covariate. For the first time, we found that GBM patients with a high level of NEK9 and PIK3CB had a greater chance of having recurrent tumors. The expression of CDCP1, IGF2R, IRAK3, LATS2, PIK3CB, ULK4, or VRK1 in primary GBM tumors was associated with recurrence-related prognosis. Notably, the level of PIK3CB in recurrent tumors was much higher than that in newly diagnosed ones. Congruent with these results, genes in the PI3K/AKT pathway showed a significantly strong correlation with recurrence rate, further highlighting the pivotal role of PIK3CB in the disease progression. Importantly, 17 SKGs together presented a novel GBM prognostic signature. SKGs identified herein are associated with recurrence rate and present prognostic significance in GBM, thereby becoming attractive therapeutic targets.

\section{INTRODUCTION}

Glioblastoma (GBM) is the most common and deadly subtype of malignant brain tumors [1]. Although the incidence of GBM is low (0.59-3.69 per 100,000 persons worldwide), its clinical outcome has been extremely poor despite aggressive upfront treatments including maximum safe surgical removal of the tumor, ionized irradiation, and chemotherapeutic treatment using temozolomide (TMZ) [2]. The five-year overall survival (OS) of GBM is approximately $4.7 \%$ in the United States
[2] and even below this rate in Europe [3]. The median survival of GBM patients receiving aforementioned concurrent therapies is only 14.6 months [4]. These grim facts therefore demonstrate an urgent need of new and effective treatments as well as powerful prognostic markers to assist these treatments for this deadly disease.

Prognosis markers are important for GBM treatments such as chemotherapy temozolomide (TMZ). For example, promoter methylation status of the O6methylguanine-DNA methyltransferase (MGMT) - an enzyme that repairs TMZ-induced DNA damage - predicts 
the response of GBM patients to this drug $[2,5,6]$. In several clinical trials, MGMT methylation was confirmed as an important prognosis marker associated with improved OS of newly diagnosed GBM patients receiving TMZ [6-12]. However, given the limited therapeutic efficacy of TMZ, the importance of MGMT as a standard prognostic marker for GBM is compromised. There are microRNAs and some other genes such as epidermal growth factor receptor (EGFR) or CD133 that show a close correlation with GBM prognosis [13-17]. However, targeting them has not yet been very successful. Recent advances in large-scale genome DNA sequencing have significantly contributed to the identification of new biomarkers for GBM [18, 19]. For instance, genetic mutations in isocitrate dehydrogenase 1 or 2 (IDH1/2) genes have shown a strong association with better outcome in a subgroup of GBM [20,21]. An inhibitor of IDH1 significantly retards GBM growth through inducing differentiation [22]. However, this genetic approach often lacks functional information of candidate genes thereby requiring a further extensive and time-consuming investigation.

Loss-of-function screening using a library of short hairpin RNAs (shRNAs) [23-29] provides a useful platform that allows a search of candidate genes for cancer therapeutic development based on their functions/ activities. Yet this approach has not been used to identify genes that can serve as both therapeutic targets and prognostic markers. In this report, we employed a loss-offunction screen using a library of shRNAs against human kinases and identified 20 kinases critical for the survival of human U87MG GBM cells. Further analyses revealed candidate kinases, whose expression correlated with the prognosis of newly diagnosed and/or recurrent GBM. In addition, we for the first time have revealed kinase genes with a strong association with recurrence rate.

\section{RESULTS}

\section{A loss-of-function screen identifies new kinases essential for the survival of human U87MG GBM cells}

To identify genes with a potential to be new biomarkers and therapeutic targets, we focused on kinase genes that often govern cancer cell survival. To identify these kinases, we employed a loss-of-function screen (outlined in Figure 1A) that utilizes a library of shRNAs targeting 781 human kinase genes. We divided the human U87MG GBM cells transduced with viruses harboring the above library of shRNAs into two parts: one part was collected immediately as passage 0 (P0); the other part continued a 7-day culture and was saved as passage 7 (P7). The genomic DNA was then isolated and DNA libraries containing PCR-amplified shRNA sequences were prepared. By using Solexa deep sequencing, we profiled shRNAs. In principle, an shRNA that causes a drastic growth inhibition would be depleted from the cell population and has a decreased sequencing read number at P7. We found that 63 shRNAs had 2-fold less of the sequencing read number at P7 compared to P0 (Figure 1B). We further validated these candidates as follows. 49 individual candidate shRNAs decreased the viability of $\mathrm{U} 87 \mathrm{MG}$ cells to $\leq 60 \%$, compared to those with the control non-silencing (NS) shRNA (Figure 1C). 23 out of 49 shRNAs caused a $\geq 2$-fold reduction of mRNAs of their target genes (Figure 1D). As there were multiple shRNAs against CSNK1E or MELK, 23 shRNAs targeted 20 kinase genes that are important for the survival of U87MG cells. Full names of 20 candidate kinase genes were listed in Table 1 . We termed these 20 candidates as survival kinase genes (SKGs). Among these SKGs, MELK has been previously reported as a survival gene in GBM [30].

\section{SKGs are enriched in GBM}

We then tested the hypothesis that SKGs are enriched in GBM due to their essentiality in GBM survival. We queried online gene expression databases such as BioGPS [31], Oncomine (ThermoFisher Scientific), and The Human Protein Atlas [32]. We found that mRNA levels of 8 SKGs (IGF2R, MAP4K3, MELK, NEK9, PFKP, STK3, TBRG4, and VRK1) were at least 1.5-fold higher in U87MG cells than those in astrocytes based on BioGPS (Figure 2A, grey bars). We also analyzed two GBM datasets (Bredel Brain \#2 and TCGA brain) in Oncomine. 17 SKGs (except IQCD, PIK3CB, and PRPSAP1) showed statistically higher levels $(P<0.05)$ of their mRNAs in GBM tissues when compared to normal brain tissues (Figure 2B, grey bars). Furthermore, we compared the protein levels of SKGs between glioma and normal brain. There were more than $8 \%$ of cases with a higher protein level of 12 SKGs (CDCP1, CDKL5, CSNK1E, IGF2R, IQCD, MAP4K3, MELK, NEK9, STK3, TBRG4, ULK4, and VRK1) in glioma (Figure 2C, grey bars). Together, these results demonstrate that SKGs are enriched in GBM.

\section{Expression of SKGs correlates with the OS of GBM patients}

The above results prompted us to evaluate the potential of SKGs as GBM prognostic markers. We retrieved the gene expression data of initial/primary GBM tumors and the corresponding patient information associated with these tumors from TCGA [33] (http:// cancergenome.nih.gov/). The MGMT mRNA level (used as a positive control) showed an inverse correlation with the OS of GBM (Figure 3A), congruent with previous reports $[6,8]$. We then analyzed 20 SKGs using the 
Kaplan Meier (KM) survival analysis. We found that the expression of CDCP1, CDKL5, LATS2, PRKAA1, STK3, and ULK4 was adversely associated with the OS of GBM and their Log-Rank $P$ values were less than 0.05 or 0.01 (Figure 3B-3G). Other SKGs showed no statistical significance (Figure $3 \mathrm{H}$ and Supplementary Figure $\mathrm{S} 1$ ). There was no expression data pertaining to CDK11B in this cohort of GBM patients (Figure $3 \mathrm{H}$ ). Patients with low levels of the above 6 SKGs lived 1.2 to 3.7 months longer than those with high levels of SKGs (Figure 3I). In contrast, patients with less MGMT lived three month longer. Hence, CDCP1, CDKL5, LATS2, PRKAA1, STK3, and ULK4 present prognostic significance in the OS of GBM.

We next utilized a Cox proportional hazard model to test whether TMZ treatment or age could be used as a covariate with SKGs to better measure GBM prognosis. TMZ and age have been associated with GBM clinical outcomes [4, 34]. The hazard ratios (HRs, which define the death risk) of SKGs with prognostic significance were equivalent to or higher than that of MGMT, whereas SKGs with no prognostic significance had a lower HRs (Table 2, Cox Univariate and highlighted in grey), verifying the results of KM survival analysis (Figure $3 \mathrm{~B}-3 \mathrm{H}$ ). When TMZ - but not age-was used as a covariate, HRs of prognostic SKGs (except CDKL5) increased (Table 2, comparing Cox Univarate with Cox Multivariate with TMZ). Intriguingly, IRAK3 and TBRG4 showed a significant inverse correlation $(P<0.05)$ with the OS of GBM only when TMZ was used as a covariate (Table 2, highlighted in grey), whereas the expression of CSNK1E was positively associated with GBM prognosis (Table 2, highlighted in grey). Thus, the Cox multivariate analysis with TMZ reveals three more SKGs with prognostic significance. However, the analysis of Cox multivariate with age revealed that only CDCP1 and CDKL5 showed statistical significance (Table 2, Cox multivariate with age). These results suggest that SKGs and TMZ (but not age) together show a better prognostic correlation.

\section{Expression of SKGs correlates with the incidence rate and prognosis of recurrent GBMs}

Nearly $90 \%$ of GBM patients experience tumor recurrence two years after treatments [35]. Often patients with a recurrent tumor can not undergo a second major
A

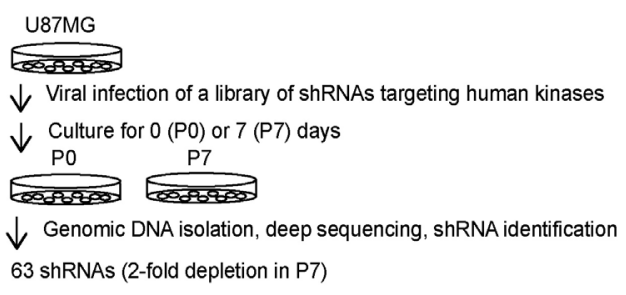

C
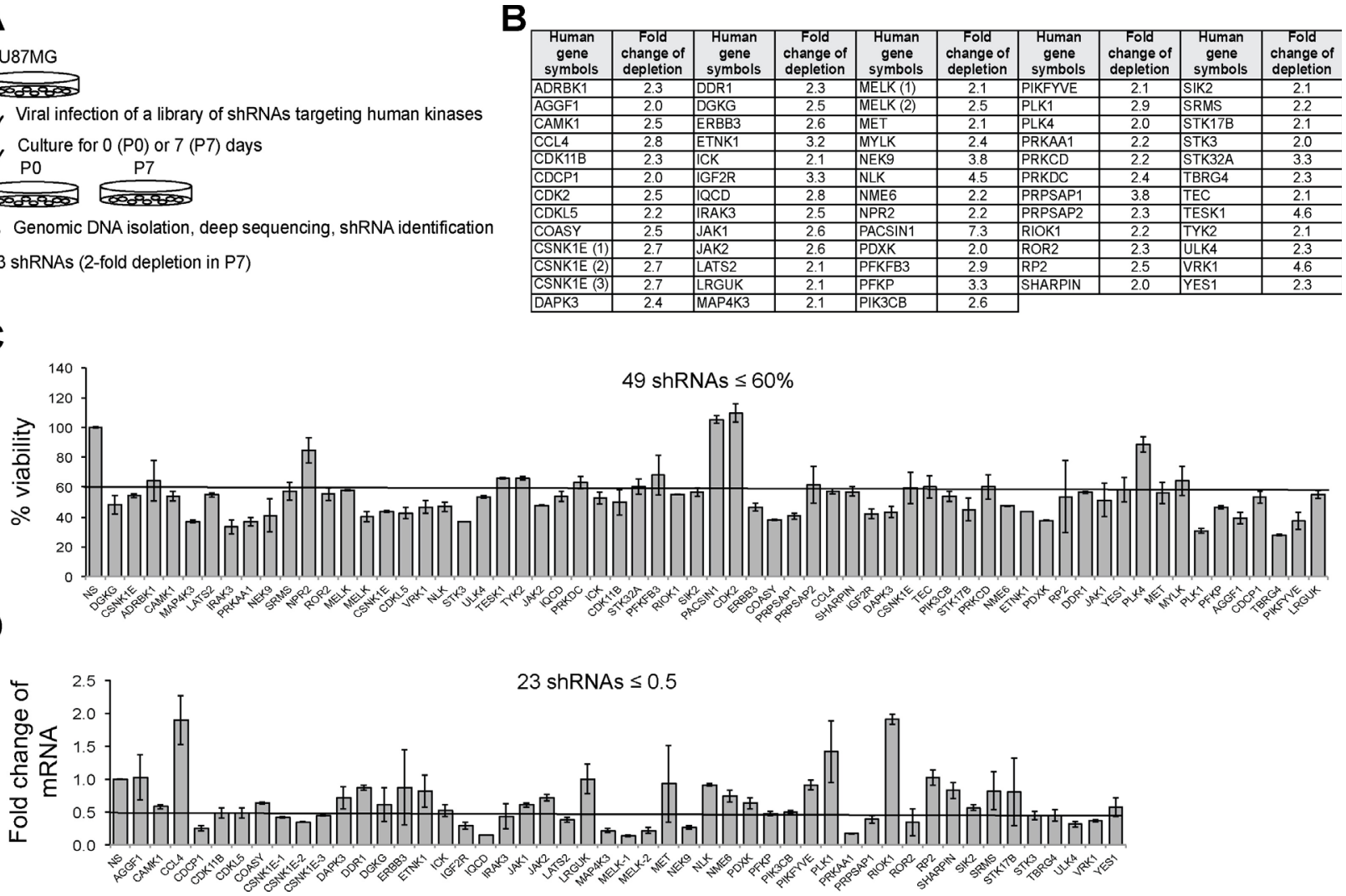

49 shRNAs $\leq 60 \%$ 
Table 1: Survival kinase genes

\begin{tabular}{|c|l|}
\hline \multicolumn{1}{|c|}{ Gene Symbol full name } \\
\hline CDCP1 & CUB Domain Containing Protein 1 \\
\hline CDK11B & Cyclin-Dependent Kinase 11B \\
\hline CDKL5 & Cyclin-Dependent Kinase-Like 5 \\
\hline CSNK1E & Casein Kinase 1, Epsilon \\
\hline IGF2R & Insulin-Like Growth Factor 2 Receptor \\
\hline IQCD & IQ Motif Containing D \\
\hline IRAK3 & Interleukin-1 Receptor-Associated Kinase 3 \\
\hline LATS2 & Large Tumor Suppressor Kinase 2 \\
\hline MAP4K3 & Mitogen-Activated Protein Kinase Kinase Kinase Kinase 3 \\
\hline MELK & Maternal Embryonic Leucine Zipper Kinase \\
\hline NEK9 & NIMA-Related Kinase 9 \\
\hline PFKP & Phosphofructokinase, Platelet \\
\hline PIK3CB & Phosphatidylinositol-4, 5-Bisphosphate 3-Kinase, Catalytic Subunit Beta \\
\hline PRKAA1 & Protein Kinase, AMP-Activated, Alpha 1 Catalytic Subunit \\
\hline PRPSAP1 & Phosphoribosyl Pyrophosphate Synthetase-Associated Protein 1 \\
\hline ROR2 & Receptor Tyrosine Kinase-Like Orphan Receptor 2 \\
\hline STK3 & Serine/Threonine Kinase 3 \\
\hline TBRG4 & Transforming Growth Factor Beta Regulator 4 \\
\hline ULK4 & Unc-51 Like Kinase 4 \\
\hline VRK1 & Vaccinia Related Kinase 1 \\
\hline
\end{tabular}

neurosurgery and resist radiation and chemotherapy [35]. Thus, identifying recurrence-related SKGs would help in the early diagnosis of and perhaps treatment for recurrence. To determine the role of SKGs in tumor recurrence, we first selected GBM patients with disease progression information including post-treatment recurrence data. Note that the sample size was small (from 17 to 33) due to the limited information regarding recurrence (Supplementary Table S1). We first analyzed the recurrence rate in GBM patients with a high or low level of SKGs. The high levels of 14 SKGs were associated with a greater incidence of recurrence (Figure 4A and Supplementary Table S1). However, only NEK9 $(P<0.01)$, PFKP $(P<0.05)$, and PIK3CB $(P<0.05)$ showed a statistically significant difference, highlighting their potential in predicting GBM recurrence rate. Further KM survival analysis revealed that the Log-Rank $P$ values of CDCP1, IGF2R, IRAK3, LATS2, PIK3CB, ULK4, and VRK1 were $<0.05$ (Figure 4B and Supplementary Figure S2), suggesting that the expression of these SGKs in the newly diagnosed GBM has a statistically significant correlation with the patient survival associated with recurrence. Notably, CDCP1, IRAK3,
LATS2 and ULK4 also presented prognostic significance in the OS of GBM (Figure 3 and Table 2). The difference in median survival time ranged from 5.2 to 11.6 months (Figure 4B), in stark contrast to the maximum 3.7 months of GBMs' OS (Figure 3I). These results demonstrate a pivotal role of SKGs in GBM recurrence. As an exception, the low level of VRK1 was associated with a shorter life span of GBM recurrence (Figure 4B). By contrast, MGMT and PTEN failed to show a significant correlation with either recurrence rate (Figure 4A and Supplementary Table S1) or recurrence-associated survival (Figure 4B and Supplementary Figure S2). Taken together, we have revealed new biomarkers for the prognosis of GBM patients with recurrent tumors.

\section{PI3K/AKT pathway is associated with the incidence rate and prognosis of GBM recurrence}

Among SKGs associated with GBM recurrence, one intriguing gene is PIK3CB, a candidate SKG that showed a strong correlation with both recurrence rate and prognosis (Figure 4A and 4B). Consistent with these results, the mRNA levels of PIK3CB were significantly 
higher $(P=0.019)$ in recurrent tumors than those in newly diagnosed GBM tumors (Figure $4 \mathrm{C}$ ). PIK3CB is a catalytic subunit of PI3K complex [36]. The PI3K/AKT pathway has been implicated in GBM recurrence and its inhibitors have been used to treat recurrent tumors [37, 38]. We then analyzed multiple kinases in or downstream of this pathway. GBM patients expressing high levels of kinases in the PI3K/AKT pathway had a greater chance of recurrence (Figure 4D, panel mRNAs). Among these genes, PIK3R1, AKT1, and AKT3 showed a statistical significance $(P<0.05)$. We also analyzed the protein levels of these kinases using the TCGA Reverse Phase Protein Array dataset. The high protein level was associated with a greater chance of tumor recurrence (Figure 4D, panel Proteins). Notably, the PI3K regulatory subunit PI3K p85 and an MTOR target $\mathrm{p} 70 \mathrm{~S} 6 \mathrm{~K}$ showed a statistical significance $(P<0.05)$. Collectively, our results demonstrate that the $\mathrm{PI} 3 \mathrm{~K} / \mathrm{AKT}$ signaling is involved in the disease progression, highlighting the importance of $\mathrm{PIK} 3 \mathrm{CB}$ as a predictor of recurrence rate and/or a prognosis marker associated with recurrence.
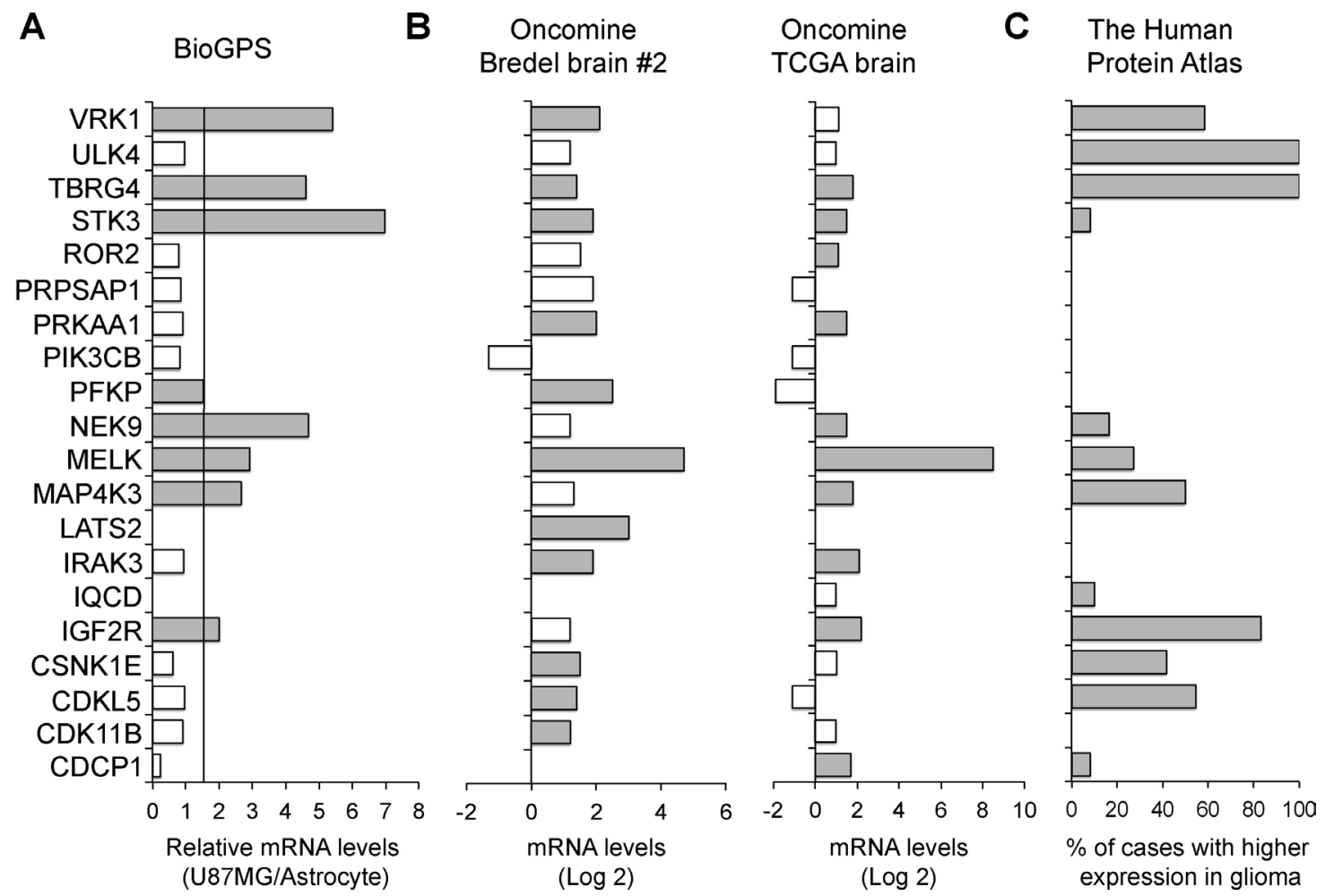

\section{A group of SKGs presents a novel prognostic signature for GBM}

To further verify the role of SKGs in GBM prognosis, we analyzed the prognostic potential of multiple SKGs as a group. We employed an online program Glioblastoma Bio Discovery Portal (GBM-BioDP) that exploits different computer algorithms to cluster genes together based on their expression profile in the TCGA GBM datasets [39]. Three SKGs (CDK11B, IQCD, and PRKAA1) were not included in this analysis due to the lack of information in all datasets. Based on the expression profile of the remaining 17 SKGs, GBM patients were divided into cluster $\mathrm{A}$ and $\mathrm{B}$ (Figure 5A). Cluster A showed a significantly shorter life span than cluster B with a Log-Rank $P$ at 0.016 (Figure 5B). The clustering analysis also identified three subgroups (Figure 5A). We then analyzed the relationship of SKGs in these subgroups with GBM survival by dividing GBM patients into clusters (Supplementary Figure S3) followed by KM survival analysis (Figure 5C and Supplementary Figure S4). None of these subgroups presented a significant correlation with

Figure 2: SKGs are enriched in GBM. (A) mRNA levels of SKGs in U87MG. Gene expression data was retrieved from the BioGPS database. Fold changes of SKG mRNAs in U87MG cells compared to those in astrocytes were shown. The cut-off line was 1.5-fold increase. SKGs with a high level of mRNA in U87MG cells were labeled as grey bars. (B) mRNA levels of SKGs in glioblastoma tissues. Gene expression data was retrieved from the Oncomine database. The mRNA levels of SKGs in glioblastoma were compared to those in normal brain tissues. Results from two studies (Bredel brain $\# 2$ and TCGA brain) were shown. ${ }^{*} P<0.05$. SKGs with a statistically significant increase of mRNA in glioblastoma in either study were labeled as grey bars. (C) Protein levels of SKGs in glioma. Protein expression data was retrieved from the Human Protein Atlas database. The percentages of glioma cases with a higher level of SKGs compared to the normal brain were shown. SKGs with more protein in glioma were labeled as grey bars. PRPSAP1 and PIK3CB showed a negative result in all these analyses. 
GBM survival. Together, our data strongly suggests that a group of 17 SKGs presents a novel signature for GBM prognosis.

\section{DISCUSSION}

The results presented herein are of importance due to their significance in measuring the prognosis of GBM and/or tumor recurrence as well as the druggablilty of these SKGs. Based on the literature (Supplementary Table $\mathrm{S} 2$ ), $10 \mathrm{SKG}$ has been reported previously as a prognosis biomarker for certain types of cancer. However, none of SKGs has been used as a prognosis marker for GBM. Our finding that SKGs can predict GBM prognosis is therefore novel and unprecedented. Interestingly, the low rather than high levels of CSNK1E, IGF2R, IRAK3, and PRKAA1 relate to poor cancer prognosis (Supplementary Table S2), suggesting that kinases often play a divergent role in different types of cancer. By contrast, the high expression levels of most prognostic SKGs (except CSNK1E) show a correlation with the poor prognosis of GBM patients (Table 2). Hence, inhibiting these prognostic SKGs represents a viable approach to developing new therapies for GBM.

Our study also reveals powerful biomarkers for the diagnosis and/or prognosis of tumor recurrence, given the fact that no similar markers have been established to date except magnetic resonance imaging (MRI) [40]. MRI is a costly approach often perplexed with pseudo-progression [41]. Measuring the gene expression levels in tumor biopsies would provide an effective and less expensive way to monitor disease progression. Particularly important is that the high levels of NEK9, PFKP, and PIK3CB in the newly diagnosed GBM tumors are associated with the greater risk of recurrence. Thus, NEK, PFKP, and PIK3CB can be used as a predictive marker for GBM recurrence

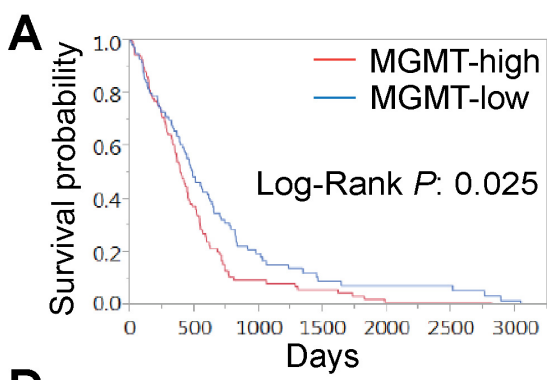

D

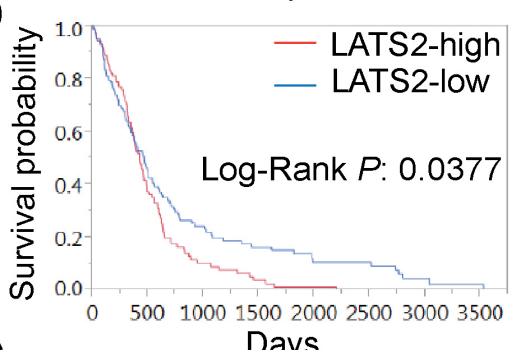

G

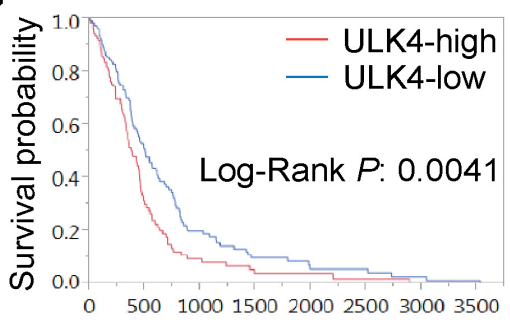

H

\begin{tabular}{|l|c|c|c|l|c|}
\hline $\begin{array}{c}\text { Gene } \\
\text { Symbol }\end{array}$ & $\begin{array}{c}\text { Log- } \\
\text { Rank } \boldsymbol{P}\end{array}$ & $\begin{array}{c}\text { Gene } \\
\text { Symbol }\end{array}$ & $\begin{array}{c}\text { Log- } \\
\text { Rank } \boldsymbol{P}\end{array}$ & $\begin{array}{c}\text { Gene } \\
\text { Symbol }\end{array}$ & $\begin{array}{c}\text { Log- } \\
\text { Rank } \boldsymbol{P}\end{array}$ \\
\hline CDK11B & No data & MAP4K3 & 0.396 & PRPSAP1 & 0.238 \\
\hline CSNK1E & 0.065 & MELK & 0.866 & ROR2 & 0.911 \\
\hline IGF2R & 0.593 & NEK9 & 1.115 & TBRG4 & 0.127 \\
\hline IQCD & 0.325 & PFKP & 0.993 & VRK1 & 0.721 \\
\hline IRAK3 & 0.112 & PIK3CB & 0.429 & \multicolumn{3}{|c}{} \\
\cline { 1 - 2 } & & &
\end{tabular}

B

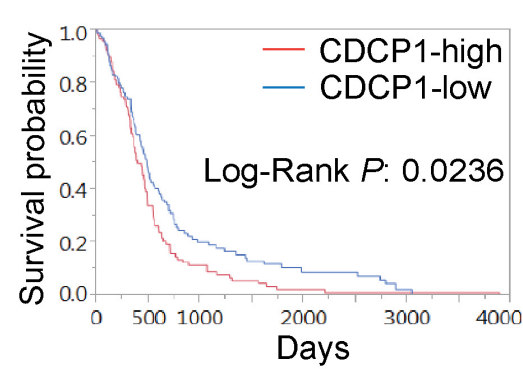

E

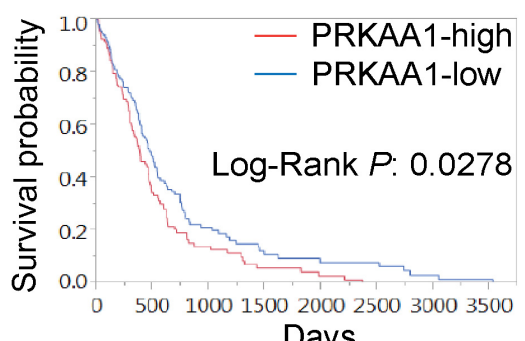

Days

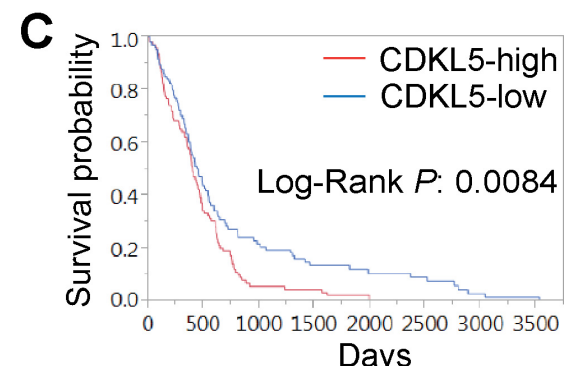

$\mathbf{F}$

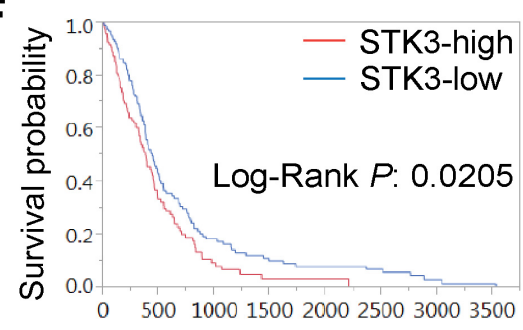

Days

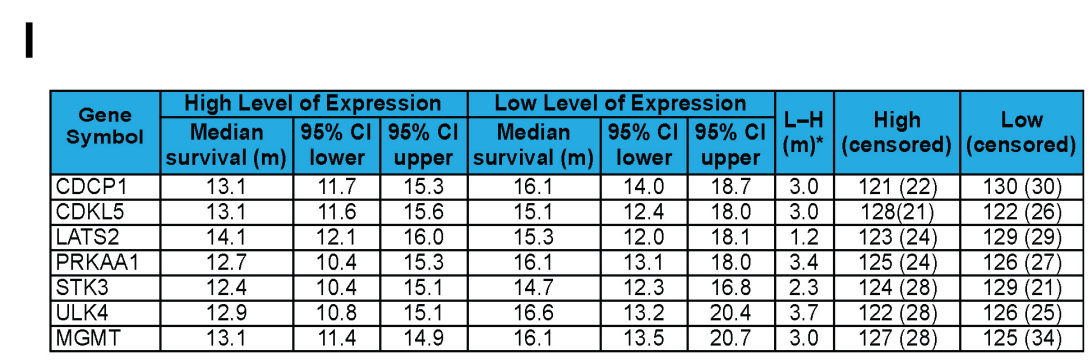

Figure 3: Expression of SKGs correlates with GBM prognosis. The Kaplan Meier analysis was performed using the TCGA GBM datasets. The survival curves of MGMT (A), CDCP1 (B), CDKL5 (C), LATS2 (D), PRKAA1 (E), STK3 (F), and ULK4 (G) with LogRank $P$ values were shown. The Log-Rank $P$ values of other SKGs that showed no statistical significance were listed in $(\mathbf{H})$. The media survival time of prognostic SKGs was shown in (I). 
Table 2: Survival analysis of SKGs using the cox proportional hazard model

\begin{tabular}{|c|c|c|c|c|c|c|c|c|c|c|c|c|}
\hline \multirow[b]{2}{*}{$\begin{array}{c}\text { Gene } \\
\text { Symbol }\end{array}$} & \multicolumn{4}{|c|}{ Cox Univariate } & \multicolumn{4}{|c|}{ Cox Multivariate with TMZ } & \multicolumn{4}{|c|}{ Cox Multivariate with Age } \\
\hline & $\begin{array}{c}\text { HR } \\
\text { (H vs } \\
\text { L) }\end{array}$ & $\begin{array}{c}95 \% \\
\text { CI } \\
\text { lower }\end{array}$ & $\begin{array}{c}95 \% \\
\text { CI } \\
\text { upper }\end{array}$ & $\begin{array}{c}\text { Log- } \\
\text { Rank } \\
p\end{array}$ & $\begin{array}{c}\text { HR } \\
\text { (H vs } \\
\text { L) }\end{array}$ & $\begin{array}{c}95 \% \\
\text { CI } \\
\text { lower }\end{array}$ & $\begin{array}{c}95 \% \\
\text { CI } \\
\text { upper }\end{array}$ & $\begin{array}{c}\text { Log- } \\
\text { Rank } \\
p\end{array}$ & $\begin{array}{c}\text { HR } \\
\text { (H } \\
\text { vs L) }\end{array}$ & $\begin{array}{c}95 \% \\
\text { CI } \\
\text { lower }\end{array}$ & $\begin{array}{c}95 \% \\
\text { CI } \\
\text { upper }\end{array}$ & $\begin{array}{c}\text { Log- } \\
\text { Rank } \\
p\end{array}$ \\
\hline CDCP1 & 1.382 & 1.042 & 1.832 & 0.025 & 1.384 & 1.040 & 1.842 & 0.026 & 1.332 & 1.006 & 1.764 & 0.045 \\
\hline CDK11B & $\begin{array}{l}\text { No } \\
\text { Data }\end{array}$ & & & & & & & & & & & \\
\hline CDKL5 & 1.467 & 1.102 & 1.957 & 0.009 & 1.441 & 1.074 & 1.935 & 0.015 & 1.368 & 1.027 & 1.827 & 0.032 \\
\hline CSNK1E & 0.762 & 0.569 & 1.017 & 0.065 & 0.684 & 0.507 & 0.921 & 0.012 & 0.954 & 0.708 & 1.282 & 0.755 \\
\hline IGF2R & 0.927 & 0.702 & 1.224 & 0.594 & 0.804 & 0.598 & 1.081 & 0.150 & 1.025 & 0.774 & 1.356 & 0.865 \\
\hline IQCD & 1.152 & 0.868 & 1.527 & 0.326 & 1.226 & 0.918 & 1.637 & 0.167 & 1.077 & 0.810 & 1.432 & 0.607 \\
\hline IRAK3 & 1.255 & 0.947 & 1.661 & 0.113 & 1.416 & 1.056 & 1.898 & 0.020 & 1.050 & 0.790 & 1.396 & 0.735 \\
\hline LATS2 & 1.356 & 1.016 & 1.810 & 0.039 & 1.411 & 1.048 & 1.903 & 0.023 & 1.057 & 0.790 & 1.417 & 0.710 \\
\hline MAP4K3 & 1.127 & 0.854 & 1.483 & 0.397 & 1.274 & 0.960 & 1.686 & 0.093 & 1.042 & 0.789 & 1.373 & 0.772 \\
\hline MELK & 1.024 & 0.775 & 1.354 & 0.866 & 1.125 & 0.843 & 1.503 & 0.425 & 0.989 & 0.748 & 1.309 & 0.941 \\
\hline NEK9 & 1.166 & 0.875 & 1.552 & 0.293 & 1.344 & 0.991 & 1.821 & 0.057 & 1.056 & 0.792 & 1.407 & 0.708 \\
\hline PFKP & 0.999 & 0.749 & 1.333 & 0.993 & 0.854 & 0.633 & 1.150 & 0.298 & 1.191 & 0.887 & 1.600 & 0.245 \\
\hline PIK3CB & 1.119 & 0.845 & 1.478 & 0.431 & 1.160 & 0.869 & 1.543 & 0.313 & 0.918 & 0.690 & 1.219 & 0.555 \\
\hline PRKAA1 & 1.372 & 1.034 & 1.822 & 0.029 & 1.499 & 1.120 & 2.006 & 0.006 & 1.227 & 0.923 & 1.633 & 0.159 \\
\hline PRPSAP1 & 1.185 & 0.893 & 1.573 & 0.239 & 1.229 & 0.916 & 1.648 & 0.169 & 1.174 & 0.884 & 1.558 & 0.266 \\
\hline ROR2 & 1.016 & 0.766 & 1.353 & 0.911 & 1.078 & 0.805 & 1.449 & 0.616 & 1.138 & 0.854 & 1.522 & 0.379 \\
\hline STK3 & 1.393 & 1.050 & 1.848 & 0.022 & 1.558 & 1.166 & 2.078 & 0.003 & 1.096 & 0.817 & 1.471 & 0.540 \\
\hline TBRG4 & 1.245 & 0.939 & 1.650 & 0.128 & 1.399 & 1.042 & 1.880 & 0.026 & 1.179 & 0.889 & 1.565 & 0.254 \\
\hline ULK4 & 1.518 & 1.139 & 2.023 & 0.005 & 1.550 & 1.153 & 2.080 & 0.004 & 1.244 & 0.926 & 1.671 & 0.147 \\
\hline VRK1 & 0.950 & 0.717 & 1.260 & 0.722 & 1.050 & 0.785 & 1.407 & 0.743 & 0.976 & 0.737 & 1.295 & 0.864 \\
\hline MGMT & 1.390 & 1.040 & 1.858 & 0.026 & 1.406 & 1.045 & 1.893 & 0.024 & 1.369 & 1.023 & 1.833 & 0.034 \\
\hline
\end{tabular}

Data was retrieved from TCGA. HR (H vs L): Hazard ratios comparing the group with high (H) levels of SKGs to the one with low (L) levels. CI: confidence interval. Log-Rank $P$ values less than 0.05 were highlighted in grey.

rate, highlighting a potentially important approach for an early diagnosis.

The identification of PIK3CB as a marker for predicting GBM recurrence rate and prognosis sets up stage for future development of diagnostic tools and therapies for this disease. In fact, targeting PI3K has been an effort in the clinic to treat GBM. However, the therapeutic benefits are limited. For instance, a recent phase II trial of PX-866 (a pan PI3K inhibitor) failed to yield promising therapeutic effects and demonstrated no association of PI3KCA with disease progression [38]. Based on our findings, it would be imperative to further investigate the possibility of targeting PIK3CB specifically, rather than the PI3K pathway that has more profound impact on cell fate. Interestingly, specific and clinically applicable inhibitors of PIK3CB (i.e. AZD8186 [42] and GSK2636771) are currently available [43], presenting a potential opportunity for clinical trials to treat newly diagnosed or recurrent GBM.

\section{METHODS}

\section{Reagents}

The Cell-Titer $96^{\circledR}$ Aqueous One solution cell proliferation assay (MTS) was purchased from Promega. The SYBER green mix was purchased from Promega. The TRC kinase shRNA gene family library was purchased from GE Dharmacon. The Column-free ${ }^{\mathrm{TM}}$ plasmid miniprep kit was purchased from Lamda Biotech, Inc. 
The QIAamp DNA extraction kit was purchased from QIAGEN. Puromycin was purchased from Thermo Fisher Scientific. Trizol and the SuperScript ${ }^{\circledR}$ III RT were purchased from Thermo Fisher Scientific.

\section{Cell culture}

U87MG cells were maintained in Dulbecco's modified Eagle medium (Thermo Fisher Scientific) supplemented with $10 \%$ fetal bovine serum (FBS) (Atlas Biologicals, Inc.), streptomycin $(100 \mu \mathrm{g} / \mathrm{ml})$ and penicillin (100 IU/ml) (Thermo Fisher Scientific). Cells were cultured in a $37^{\circ} \mathrm{C}$ incubator with $5 \% \mathrm{CO}_{2}$.

\section{shRNA library preparation}

4,518 shRNA constructs (against 781 human kinases) were maintained as a single clone of bacteria in glycerol stock in 96-well plates. To prepare a mix of plasmids, each bacterial plate was replicated in another 96-well culture plate with $1.8 \mathrm{ml}$ of $2 \times \mathrm{LB}$ in each well. Plates were then incubated at $37^{\circ} \mathrm{C}$ with vigorous shaking for 24 hours. Plasmids were then prepared using a miniprep kit adapted for 96-well plate. The concentration of each plasmid was determined using a nanodrop (Thermo Fisher Scientific). Equal amount of $\sim 450$ plasmids was mixed together as a single plasmid pool. The entire library was then divided into 10 pools.

\section{Lentivirus preparation}

The TRC shRNAs are built upon pLKO.1 vector that can be used to generate lentivirus. A pool of plasmids of the TRC kinase shRNA library was transfected into HEK293T cells together with packaging plasmids pMD2.g and psPax2. 48 hours later, the culture media that contained lentiviruses was collected and divided into small aliquots. Aliquots were stored at $-80^{\circ} \mathrm{C}$ freezer. The virus titer was then determined using the serial dilution assay.

\section{Loss-of-function screen for SKGs}

U87MG $\left(1 \times 10^{6}\right)$ cells were transduced with 10 pools of lentiviruses that harbor the TRC kinase shRNAs.
A

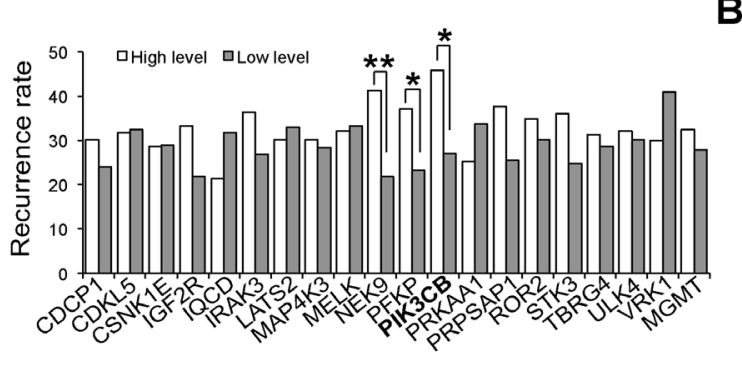

C

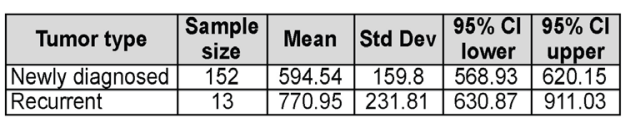

B

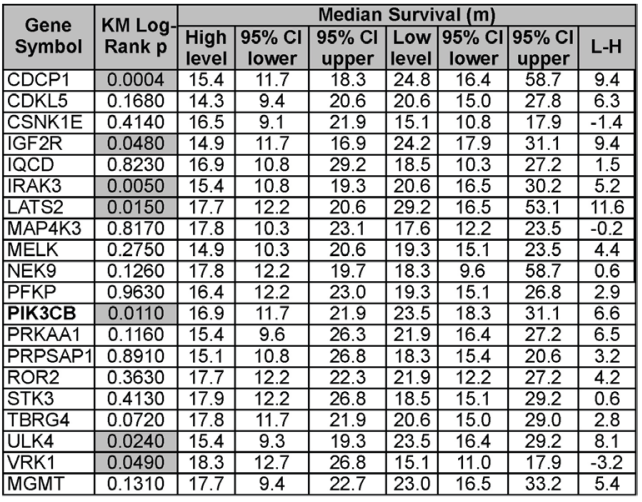

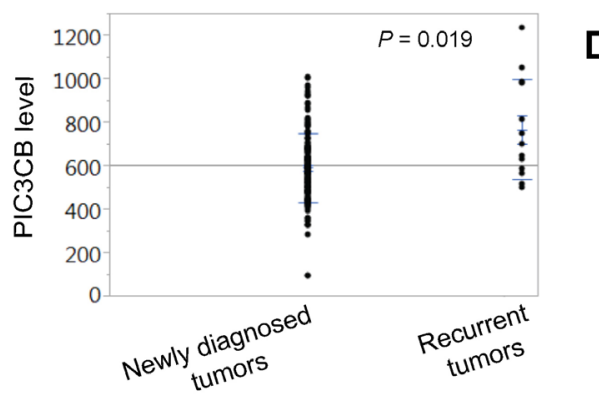

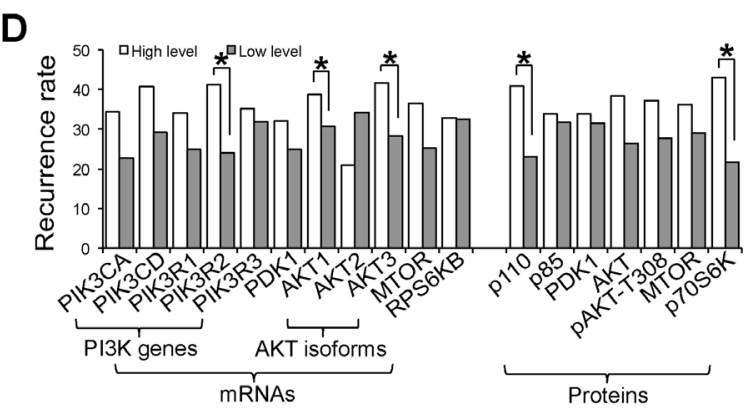

Figure 4: Expression of SKGs correlates with the incidence rate and prognosis of recurrent GBMs. (A) Recurrence rate. GBM recurrence rates in patients with a high level (white bars) or a low level of SKGs (grey bars) were shown. The statistical difference between two groups was determined by the Fisher's exact test. (B) KM survival analysis. The relationship of SKGs with the prognosis of GBMs patients with recurrent tumors was analyzed. The KM Log-Rank $P$ values were shown. The Log-Rank $P$ values less than 0.05 were highlighted in grey. VRK1 showed an effect on patient survival opposite to that exhibited by other SKGs. Median survival time of high- or low-level groups together with the lower or upper 95\% CI (confidence interval) was listed. (C) Expression of PIK3CB in newly diagnosed and recurrent GBM tumors. RNA-seq data were retrieved from the TCGA database. The mean values of PIK3CB transcripts were shown. (D) GBM recurrence and PI3K/AKT pathway. Kinases involved in or downstream of PI3K/AKT pathway were analyzed. $* P<0.05$; $* * P<0.01$. 
One day after viral infection, half of the infected cells $\left(5 \times 10^{5}\right)$ was collected as P0 (initial time point) and subjected to genomic DNA isolation described below. The other half of infected cells was cultured for another 7 days in the media containing $0.5 \mu \mathrm{g} / \mathrm{ml}$ puromycin and collected as P7 (end time point). Genomic DNA of cells at P0 and P7 was isolated using the QIAamp DNA extraction kit. shRNAs were then amplified by PCR (MF18 (5'-tacgatacaaggetgttagagag-3') and MF19 $\left(5^{\prime}\right.$-cgaaccgcaaggaaccttc- $\left.\left.3^{\prime}\right)\right)$ and sequenced using the Solexa deep sequencing. The sequencing read number of each shRNA at P0 was divided by that at P7. The shRNAs with a 2-fold less of this number were considered as candidate SKGs.

\section{Cell viability assay}

To validate the primary candidates identified from the above screen, U87MG cells were transduced with viruses of NS or individual candidate shRNAs. Cells were then selected with puromycin $(0.5 \mu \mathrm{g} / \mathrm{ml})$ for a week. Cell viability was determined using the MTS assay described previously $[44,45]$.

\section{Quantitative RT-PCR}

Quantitative RT-PCR was preformed as described previously [44, 45]. U87MG cells transduced with NS or candidate shRNAs were subject total RNA extraction using Trizol. RNA was quantified using a nanodrop. $2 \mu \mathrm{g}$ of total RNA was used to prepare cDNA using the SuperScript ${ }^{\circledR}$ III RT. mRNA levels of shRNA-targeting kinase genes were quantified using a real-time PCR assay.

\section{Analysis of gene expression}

The expression data of 20 SKGs was retrieved from BioGPS, Oncomine, and The Human Protein Atlas. For the data from BioGPS, the relative level of each SKG mRNA in GBM cell lines was obtained by dividing the arbitrary number of SKGs in tumor cell lines with that in astrocytes. The fold changes of each SKG in normal brain tissues

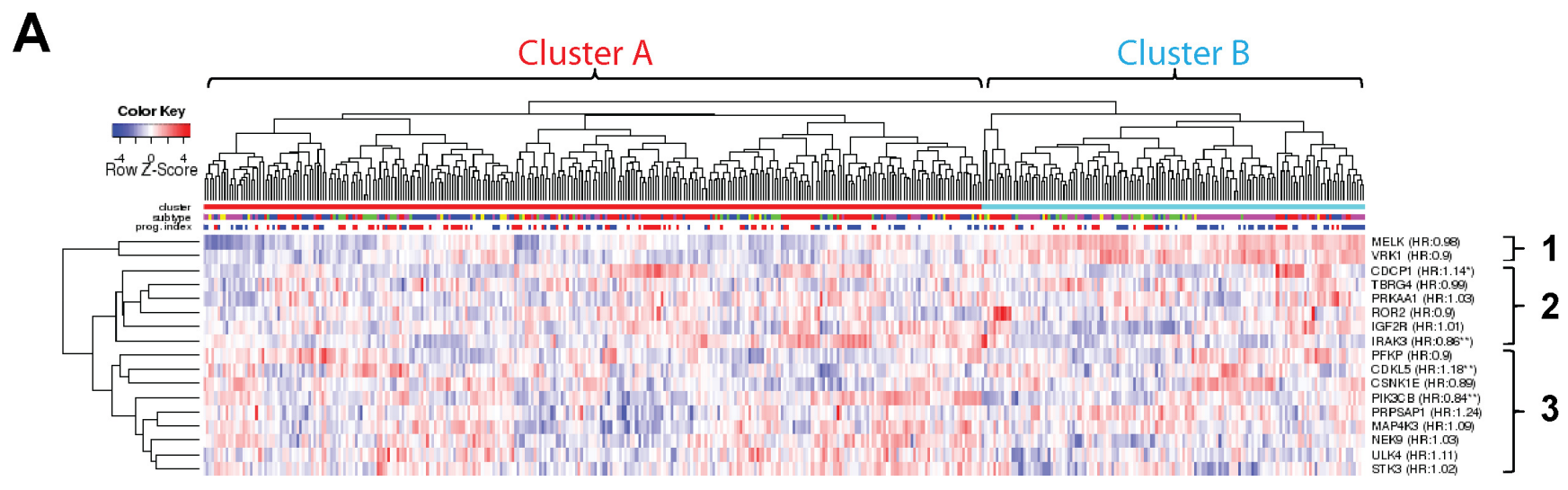

B

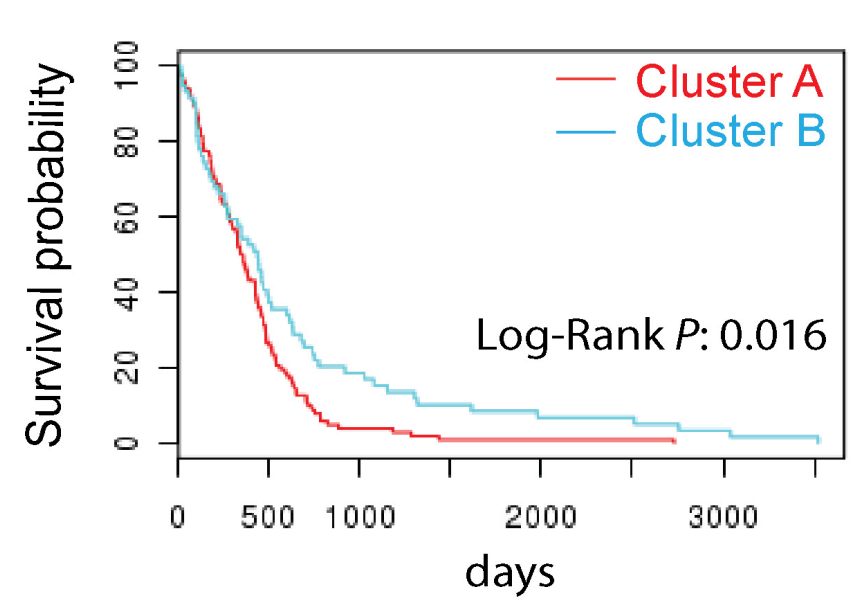

C

\begin{tabular}{|l|c|}
\hline \multicolumn{1}{|c|}{ Groups } & $\boldsymbol{P}$ values (Log-Rank) \\
\hline Group 1 & 0.639 \\
Group 2 & 0.655 \\
Group 3 & 0.051 \\
Groups 1 and 2 & 0.173 \\
Groups 2 and 3 & 0.483 \\
Groups 1 and 3 & 0.058 \\
\hline
\end{tabular}

Figure 5: A group of SKGs presents a novel prognostic signature for GBM. (A) GBM patient clustering using the GBM Bio Discovery Portal. (B) KM survival analysis. (C) Log-Rank $P$ values of KM survival analysis of different subgroups or combinations were listed. 
or glioblastoma were retrieved from Oncomine. The $p$ values determining the difference of SKG levels between normal brain and glioblastoma were also retrieved from the database. To analyze the protein expression data from the Human Protein Atlas, the level of SKG proteins (low, medium, high) in normal cerebral cortex was used as the reference. Glioma samples with the SKG protein level higher than the reference were scored. The enrichment of SKG protein in glioma was presented as percentages of glioma cases with a higher level of SKG protein.

\section{GBM patient survival analyses}

GBM patient survival analyses were performed as previously described [31]. In brief, clinical variables of glioblastoma patients, such as survival time, vital status, disease progression, TMZ treatment, and tumor recurrence, were retrieved from the TCGA (The Cancer Genome Atlas) Data Portal (https://tcga-data.nci.nih.gov/ tcga/). Gene expression data, including for glioblastoma patients (AgilentG4502A071; AgilentG4502A072), was downloaded from the Pan-cancer project (syn1461183) on Synapse (http://www.synapse.org). GBM patients were divided into high level (top 25\%) and low level (bottom $25 \%$ ) based on the SKG mRNA levels. A Kaplan Meier analysis or a Cox proportional hazard model was performed using the JMP software (SAS Institute Inc.). Recurrent tumor rates were predicted using Contingency Analysis and Fisher's Exact Test was performed using the JMP software.

\section{GBM discovery bio portal}

To analyze the expression of SKGs as groups, we employed the online program GBM Discovery Bio Portal that utilizes the same TCGA GBM datasets described above. The algorithms used to cluster GBM patients include: [1] The optimal number of clusters (NbClust); [2] Expression levels in GBM subgroups; [3] Prognostic Index, obtained by computing weighted averages of expression values with regression coefficients of a multigene Cox proportional hazards model. The datasets used herein include: [1] AgilentG4502A_07 from University of North Carolina, [2] HT_HG-U133A from Broad Institute, and [3] HuEx-1_0-st-v2 from the Berkeley Lab.

\section{ACKNOWLEDGMENTS AND FUNDING}

The authors thank all of the members of the Sheng, Kelly, and Franck's laboratories for advice and guidance of this project.

\section{GRANT SUPPORT}

This work is supported by the start-up fund from VTCRI and the research grant from The Elsa U. Pardee Foundation to Z.S.

\section{CONFLICTS OF INTEREST}

None.

\section{Authors' contributions}

Conception and design: Z. Sheng, R.T. Varghese. Development of methodology: Z. Sheng, R.T. Varghese, Y. Liang, D.F. Kelly. Analysis and interpretation of data (e.g. statistical analysis, biostatistics, computational analysis): T. Guan, C. Franck. Writing, review, and/or revision of the manuscript: Z. Sheng, R.T. Varghese.

\section{REFERENCES}

1. Ostrom QT, Gittleman H, Farah P, Ondracek A, Chen Y, Wolinsky Y, Stroup NE, Kruchko C, Barnholtz-Sloan JS. CBTRUS statistical report: Primary brain and central nervous system tumors diagnosed in the United States in 2006-2010. Neuro-oncology. 2013; 15 Suppl 2:ii1-56.

2. Ostrom QT, Bauchet L, Davis FG, Deltour I, Fisher JL, Langer CE, Pekmezci M, Schwartzbaum JA, Turner MC, Walsh KM, Wrensch MR, Barnholtz-Sloan JS. The epidemiology of glioma in adults: a "state of the science" review. Neuro-oncology. 2014; 16:896-913.

3. Sant M, Minicozzi P, Lagorio S, Borge Johannesen T, Marcos-Gragera R, Francisci S, Group EW. Survival of European patients with central nervous system tumors. Int J Cancer. 2012; 131:173-85.

4. Stupp R, Mason WP, van den Bent MJ, Weller M, Fisher B, Taphoorn MJ, Belanger K, Brandes AA, Marosi C, Bogdahn U, Curschmann J, Janzer RC, Ludwin SK, et al. Radiotherapy plus concomitant and adjuvant temozolomide for glioblastoma. N Engl J Med. 2005; 352:987-96.

5. Hegi ME, Diserens AC, Godard S, Dietrich PY, Regli L, Ostermann S, Otten P, Van Melle G, de Tribolet N, Stupp R. Clinical trial substantiates the predictive value of O-6methylguanine-DNAmethyltransferasepromotermethylation in glioblastoma patients treated with temozolomide. Clinical cancer research. 2004; 10:1871-4.

6. Chen Y, Hu F, Zhou Y, Chen W, Shao H, Zhang Y. MGMT promoter methylation and glioblastoma prognosis: a systematic review and meta-analysis. Archives of medical research. 2013; 44:281-90.

7. Lalezari S, Chou AP, Tran A, Solis OE, Khanlou N, Chen W, Carrillo JA, Chowdhury R, Selfridge J, Sanchez DE, Wilson RW, Zurayk M, Lalezari J, et al. Combined analysis of O6-methylguanine-DNA methyltransferase protein expression and promoter methylation provides optimized prognostication of glioblastoma outcome. Neuro-oncology. $2013 ; 15: 370-81$.

8. Hegi ME, Liu L, Herman JG, Stupp R, Wick W, Weller M, Mehta MP, Gilbert MR. Correlation of O6-methylguanine methyltransferase (MGMT) promoter methylation with clinical outcomes in glioblastoma and clinical strategies 
to modulate MGMT activity. Journal of clinical oncology. 2008; 26:4189-99.

9. Donson AM, Addo-Yobo SO, Handler MH, Gore L, Foreman NK. MGMT promoter methylation correlates with survival benefit and sensitivity to temozolomide in pediatric glioblastoma. Pediatr Blood Cancer. 2007; 48:403-7.

10. Ishii D, Natsume A, Wakabayashi T, Hatano H, Asano Y, Takeuchi H, Shimato S, Ito M, Fujii M, Yoshida J. Efficacy of temozolomide is correlated with $1 \mathrm{p}$ loss and methylation of the deoxyribonucleic acid repair gene MGMT in malignant gliomas. Neurol Med Chir (Tokyo). 2007; 47:341-9; discussion 50.

11. Wiewrodt D, Nagel G, Dreimuller N, Hundsberger T, Perneczky A, Kaina B. MGMT in primary and recurrent human glioblastomas after radiation and chemotherapy and comparison with p53 status and clinical outcome. Int J Cancer. 2008; 122:1391-9.

12. van Nifterik KA, van den Berg J, van der Meide WF, Ameziane N, Wedekind LE, Steenbergen RD, Leenstra S, Lafleur MV, Slotman BJ, Stalpers LJ, Sminia P. Absence of the MGMT protein as well as methylation of the MGMT promoter predict the sensitivity for temozolomide. British journal of cancer. 2010; 103:29-35.

13. Zhang W, Chen H, Lv S, Yang H. High CD133 Expression Is Associated with Worse Prognosis in Patients with Glioblastoma. Mol Neurobiol. 2015 May 17; PMID: 25983032.

14. Pointer KB, Clark PA, Zorniak M, Alrfaei BM, Kuo JS, Glioblastoma cancer stem cells: Biomarker and therapeutic advances. Neurochemistry international. 2014; 71:1-7.

15. Zhang W, Zhang J, Yan W, You G, Bao Z, Li S, Kang C, Jiang C, You Y, Zhang Y, Chen CC, Song SW, Jiang T. Whole-genome microRNA expression profiling identifies a 5-microRNA signature as a prognostic biomarker in Chinese patients with primary glioblastoma multiforme. Cancer. 2013; 119:814-24.

16. Nakata S, Campos B, Bageritz J, Bermejo JL, Becker N, Engel F, Acker T, Momma S, Herold-Mende C, Lichter P, Radlwimmer B, Goidts V. LGR5 is a marker of poor prognosis in glioblastoma and is required for survival of brain cancer stem-like cells. Brain pathology. 2013; 23:60-72.

17. Ilhan-Mutlu A, Wagner L, Wohrer A, Furtner J, Widhalm G, Marosi C , Preusser M. Plasma MicroRNA-21 concentration may be a useful biomarker in glioblastoma patients. Cancer investigation. 2012; 30:615-21.

18. Parsons DW, Jones S, Zhang X, Lin JC, Leary RJ, Angenendt P, Mankoo P, Carter H, Siu IM, Gallia GL, Olivi A, McLendon R, Rasheed BA, et al. An integrated genomic analysis of human glioblastoma multiforme. Science. 2008; 321:1807-12.

19. Cancer Genome Atlas Research N. Comprehensive genomic characterization defines human glioblastoma genes and core pathways. Nature. 2008; 455:1061-8.

20. Beiko J, Suki D, Hess KR, Fox BD, Cheung V, Cabral M, Shonka N, Gilbert MR, Sawaya R, Prabhu SS, Weinberg J,
Lang FF, Aldape KD, et al. IDH1 mutant malignant astrocytomas are more amenable to surgical resection and have a survival benefit associated with maximal surgical resection. Neuro-oncology. 2014; 16:81-91.

21. Labussiere M, Sanson M, Idbaih A, Delattre JY. IDH1 gene mutations: a new paradigm in glioma prognosis and therapy? The oncologist. 2010; 15:196-9.

22. Rohle D, Popovici-Muller J, Palaskas N, Turcan S, Grommes C, Campos C, Tsoi J, Clark O, Oldrini B, Komisopoulou E, Kunii K, Pedraza A, Schalm S, et al. An inhibitor of mutant IDH1 delays growth and promotes differentiation of glioma cells. Science. 2013; 340:626-30.

23. Sa JK, Yoon Y, Kim M, Kim Y, Cho HJ, Lee JK, Kim GS, Han S, Kim WJ, Shin YJ, Joo KM, Paddison PJ, Ishitani T, et al. In vivo RNAi screen identifies NLK as a negative regulator of mesenchymal activity in Glioblastoma. Oncotarget. 2015; 6:20145-59. doi: 10.18632/oncotarget.3980.

24. Cheng P, Phillips E, Kim SH, Taylor D, Hielscher T, Puccio L, Hjelmeland AB, Lichter P, Nakano I, Goidts V. Kinome-wide shRNA screen identifies the receptor tyrosine kinase AXL as a key regulator for mesenchymal glioblastoma stem-like cells. Stem cell reports. 2015; 4:899-913.

25. Yang J, Fan J, Li Y, Li F, Chen P, Fan Y, Xia X, Wong ST. Genome-wide RNAi screening identifies genes inhibiting the migration of glioblastoma cells. PloS one. 2013; 8:e61915.

26. Tandle AT, Kramp T, Kil WJ, Halthore A, Gehlhaus K, Shankavaram U, Tofilon PJ, Caplen NJ, Camphausen K. Inhibition of polo-like kinase 1 in glioblastoma multiforme induces mitotic catastrophe and enhances radiosensitisation. European journal of cancer. 2013; 49:3020-8.

27. Gargiulo G, Cesaroni M, Serresi M, de Vries N, Hulsman D, Bruggeman SW, Lancini C, van Lohuizen M. In vivo RNAi screen for BMI1 targets identifies TGF-beta/BMP-ER stress pathways as key regulators of neural- and malignant gliomastem cell homeostasis. Cancer cell. 2013; 23:660-76.

28. Goidts V, Bageritz J, Puccio L, Nakata S, Zapatka M, Barbus S, Toedt G, Campos B, Korshunov A, Momma S, Van Schaftingen E, Reifenberger G, Herold-Mende C, et al. RNAi screening in glioma stem-like cells identifies PFKFB4 as a key molecule important for cancer cell survival. Oncogene. 2012; 31:3235-43.

29. Sheng Z, Li L, Zhu LJ, Smith TW, Demers A, Ross AH, Moser RP, Green MR. A genome-wide RNA interference screen reveals an essential CREB3L2-ATF5-MCL1 survival pathway in malignant glioma with therapeutic implications. Nature medicine. 2010; 16:671-7.

30. Nakano I, Masterman-Smith M, Saigusa K, Paucar AA, Horvath S, Shoemaker L, Watanabe M, Negro A, Bajpai R, Howes A, Lelievre V, Waschek JA, Lazareff JA, et al. Maternal embryonic leucine zipper kinase is a key regulator of the proliferation of malignant brain tumors, including brain tumor stem cells. J Neurosci Res. 2008; 86:48-60.

31. Wu C, Orozco C, Boyer J, Leglise M, Goodale J, Batalov S, Hodge CL, Haase J, Janes J, Huss JW, 3rd, Su AI. BioGPS: 
an extensible and customizable portal for querying and organizing gene annotation resources. Genome Biol. 2009; 10:R130.

32. Uhlen M, Oksvold P, Fagerberg L, Lundberg E, Jonasson K, Forsberg M, Zwahlen M, Kampf C, Wester K, Hober S, Wernerus H, Bjorling L, Ponten F. Towards a knowledgebased Human Protein Atlas. Nature biotechnology. 2010; 28:1248-50.

33. Omberg L, Ellrott K, Yuan Y, Kandoth C, Wong C, Kellen MR, Friend SH, Stuart J, Liang H, Margolin AA. Enabling transparent and collaborative computational analysis of 12 tumor types within The Cancer Genome Atlas. Nature genetics. 2013; 45:1121-6.

34. Burger PC, Green SB. Patient age, histologic features, and length of survival in patients with glioblastoma multiforme. Cancer. 1987; 59:1617-25.

35. Weller M, Cloughesy T, Perry JR, Wick W. Standards of care for treatment of recurrent glioblastoma - are we there yet? Neuro-oncology. 2013; 15:4-27.

36. Maira SM, Voliva C, Garcia-Echeverria C. Class IA phosphatidylinositol 3-kinase: from their biologic implication in human cancers to drug discovery. Expert opinion on therapeutic targets. 2008; 12:223-38.

37. Joy A, Ramesh A, Smirnov I, Reiser M, Misra A, Shapiro WR, Mills GB, Kim S, Feuerstein BG. AKT pathway genes define 5 prognostic subgroups in glioblastoma. PLoS One. 2014; 9:e100827.

38. Pitz MW, Eisenhauer EA, MacNeil MV, Thiessen B, Easaw JC, Macdonald DR, Eisenstat DD, Kakumanu AS, Salim M, Chalchal H, Squire J, Tsao MS, Kamel-Reid S, et al. Phase II study of PX-866 in recurrent glioblastoma. Neuro-oncology. 2015; 17:1270-4.

39. Celiku O, Johnson S, Zhao S, Camphausen K, ShankavaramU. Visualizing molecular profiles of glioblastoma with GBMBioDP. PLoS One. 2014; 9:e101239.
40. Boxerman JL, Zhang Z, Safriel Y, Larvie M, Snyder BS, Jain R, Chi TL, Sorensen AG, Gilbert MR, Barboriak DP. Early post-bevacizumab progression on contrast-enhanced MRI as a prognostic marker for overall survival in recurrent glioblastoma: results from the ACRIN 6677/RTOG 0625 Central Reader Study. Neuro Oncol. 2013; 15:945-54.

41. Chamberlain MC. Pseudoprogression in glioblastoma. Journal of clinical oncology : official journal of the American Society of Clinical Oncology. 2008; 26:4359; author reply -60 .

42. Hancox U, Cosulich S, Hanson L, Trigwell C, Lenaghan C, Ellston R, Dry H, Crafter C, Barlaam B, Fitzek M, Smith PD, Ogilvie D, D'Cruz C, et al. Inhibition of PI3Kbeta signaling with AZD8186 inhibits growth of PTEN-deficient breast and prostate tumors alone and in combination with docetaxel. Mol Cancer Ther. 2015; 14:48-58.

43. Sanchez RM, Erhard K, Hardwicke MA, Lin H, McSurdyFreed J, Plant R, Raha K, Rominger CM, Schaber MD, Spengler MD, Moore ML, Yu H, Luengo JI, et al. Synthesis and structure-activity relationships of 1,2,4-triazolo $[1,5-\mathrm{a}]$ pyrimidin-7 $(3 \mathrm{H})$-ones as novel series of potent beta isoform selective phosphatidylinositol 3-kinase inhibitors. Bioorganic \& medicinal chemistry letters. 2012; 22: 3198-202.

44. Guo S, Liang Y, Murphy SF, Huang A, Shen H, Kelly DF, Sobrado P, Sheng Z. A rapid and high content assay that measures cyto-ID-stained autophagic compartments and estimates autophagy flux with potential clinical applications. Autophagy. 2015; 11:560-72.

45. Murphy SF, Varghese RT, Lamouille S, Guo S, Pridham KJ, Kanabur P, Osimani AM, Sharma S, Jourdan J, Rodgers CM, Simonds GR, Gourdie RG, Sheng Z. Connexin 43 inhibition sensitizes chemoresistant glioblastoma cells to temozolomide. Cancer Res. 2015; 76:139-49. 